\title{
Experimental analysis on friction increment of surface textured flexible couples
}

\author{
Xuli ZHU*, Zhongling LIU*, Daoxin SUN*, Zixin ZHANG* and Guirong TENG** \\ ${ }^{*}$ College of Mechanical and Electronic Engineering \\ Shandong University of Science and Technology, Qingdao 266590, China \\ **Department of Mechanics \\ Shandong University of Science and Technology, Qingdao 266590, China \\ E-mail: tgrzxl@sina.com
}

Received: 17 February 2021; Revised: 22 April 2021; Accepted: 19 July 2021

\begin{abstract}
The apparent friction force of spherical convex textured rigid bodies sliding on soft materials was tested and analyzed in order to develop the technology of friction increment for frictional transmission. The rigid bodies were made of Polylactic Acid (PLA) by 3D printing, and the counterparts were made of Ethylene Propylene Diene Monomer (EPDM). Experimental results show that the spherical convex surface texture increased the friction coefficient effectively compared to that of non-textured couples. The parameters include the radius of the texture, the hardness of the soft material and the normal load play important roles in the friction increment. The mechanism of the friction increment was analyzed. The apparent friction force consists of sliding friction force and ploughing force. The ploughing force which induced by the embedment of the spherical convex texture in the soft material depends on the normal force and therefore has influence on the equivalent friction coefficient. The effective angle and embedment number were proposed to model the contact state of the friction couples. The nonlinear dependence of the embedment number on the parameters of spherical convex textures and the properties of the soft materials was analyzed. The summarized model based on the experimental result can be used to predict the equivalent friction coefficient.
\end{abstract}

Keywords : Flexible friction couple, Spherical convex texture, Friction increment, Equivalent friction coefficient, Ploughing force, Embedment number

\section{Introduction}

Surface textures can improve the properties of friction couples effectively (Etsion, I., 2005; Miyanaga, N., et al., 2020; Segu, D. Z., et al., 2015). Researches on friction performances of surface textures were mostly based on micro-textures to explore the friction mechanisms (Mukhopadhyay, A., 2016; Shimizu, M., et al., 2015; Mielonen, K., et al., 2019). In dry sliding condition, the influences of surface textures are (a) during the friction process, grooves can store friction debris from the contact surfaces, reducing the friction components of ploughing and deformation (Varenberg, M., et al., 2002; Li, J., et al., 2018); (b) on the surface of the soft material with higher viscosity, surface texture can change the contact area between two friction surfaces and change the friction force (Xiao, L., et al., 2008; Voyer, J., et al., 2019); and (c) the ploughing force produced by the protrusion on the surface is the main factor that constitutes to the apparent friction force (Hol, J., et al., 2012; Elwasli, F., et al, 2018).

For friction couples consisting of two surfaces with a vast difference in hardness, the ploughing force depends mainly on the characteristics of the harder material (Menezes, P. L., et al., 2011). In the friction process, most debris between flexible friction couples come from the soft material and have not important effect on the friction force, so that the surface texture should be prepared on the surface of the rigid bodies. Surface textures can be the array of pits or protrusions. The change of stress around pits is smaller than that of protrusions because that the nominal pressure of the couples is low, thus the protrusion texture is more suitable than pit texture for the purpose of friction increment. 
Most of the researches on friction of textures referred above focused on metal friction pairs and aimed at reducing friction coefficient. Flexible friction couples composed of a rigid body and a counterpart made of soft materials have wide application prospects in industry, especially in friction transmission and braking, e.g. drum and belt transmission of conveyors, tire and rail transmission of monorail locomotives. The references provide good ideas on hardness matching and texture distribution, but the tribological mechanism and characteristics of flexible friction couples need to be studied further, especially the friction enhancement. Increment of the friction coefficient between these couples can ensure the safety and reduce the operation costs of the friction transmissions. In this study, rigid bodies with millimeter-scale spherical convex textures were manufactured to simulate the industrial working condition better. The apparent friction force of surface textured flexible friction couples was measured. The mechanism of friction increment was analyzed, and the modeling was summarized. The results are expected to develop the design method of engineering flexible friction transmission.

\section{Experiments}

\subsection{Preparation of samples}

Several kinds of protrusions on rigid bodies were designed. The frictional states of the flexible friction couples were simulated and analyzed to investigate the stress and displacement distributions of the soft materials with different hardness. Furthermore, in order to simplify the preparation process and the analysis model, the spherical protrusion was selected as the shape of protrusions at last.

The rigid parts were printed by a 3D printer, and the printing material was Polylactic Acid (PLA). The dimensions of the samples are shown in Fig. 1, the spherical convex textures with different radius $(R=0.7,1.1,1.5 \mathrm{~mm})$, and also the smooth samples were prepared.

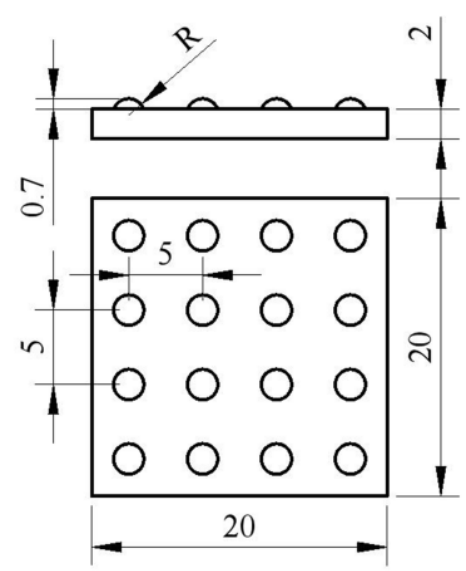

Fig. 1 Dimensions of the hard parts with spherical convex texture surface

Ethylene Propylene Diene Monomer (EPDM) was used as soft material in the experiments. EPDM samples with different hardness were obtained by different formulations. Two kinds of EPDM samples with different hardness were synthesized, and the properties of the samples are shown in Table 1.

Table 1 Properties of EPDM samples

\begin{tabular}{c|c|c|c}
\hline \hline Thickness $(\mathrm{mm})$ & $\begin{array}{c}\text { Shore hardness } \\
(\mathrm{HA})\end{array}$ & $\begin{array}{c}\text { Elastic Modulus } \\
(\mathrm{MPa})\end{array}$ & Poisson's ratio \\
\hline 3 & 57 & 3.20 & 0.47 \\
\hline 3 & 70 & 5.54 & 0.45 \\
\hline
\end{tabular}

\subsection{Friction experiments}

The experiments were carried out on the self-made equipment. The equipment consists of a mechanical device and 
a sensor signal processing system. The mechanical device includes a stepper motor, a lead screw, a slide plat and a load box with a mass block inside, as shown in Fig. 2. The spindle of the stepper motor is connected to the lead screw, the lead screw mesh with the internal thread of the slide plat, and then stepper motor can drive the slide plat moving slowly. A PLA rigid body and a piece of EPDM soft rubber were fixed to the bottom surface of the load box and the surface of the slide plat respectively. The load box was placed above the slide plat and the textured surface of the rigid body contacted with the soft material.

The sensor signal processing system includes a force sensor, a converter, a data acquisition card and a computer. The force sensor, which can also be seen in Fig. 2, was installed between the load box and the fixed support frame of the mechanical device to measure the apparent friction force. The sensor was installed as low as possible to be close to the friction surface and the mass block was placed close to the sensor according to the friction force tested in advance. The moment induced by the force of the sensor on the box was balanced with the moment induced by the gravity of the box and the mass block, so that the pressure on the friction surface was evenly distributed The converter converted the force signal of the force sensor into an electrical signal and transmitted the data to the computer through the data acquisition board.

The slide plat was set to move at a velocity of $5 \mathrm{~mm} / \mathrm{s}$ by controlling the rotating speed of the motor. The force measured by the sensor was equal to the apparent friction force because that the contact surfaces were horizontal during friction processes. The mass of the load box attached with a rigid body was $0.05 \mathrm{~kg}$. The minimum mass of the mass blocks was $0.1 \mathrm{~kg}$ and the maximum mass was $1 \mathrm{~kg}$. The total weight of the box and the mass blocks inside was the normal force acting on the contacting surfaces of the testing couple.

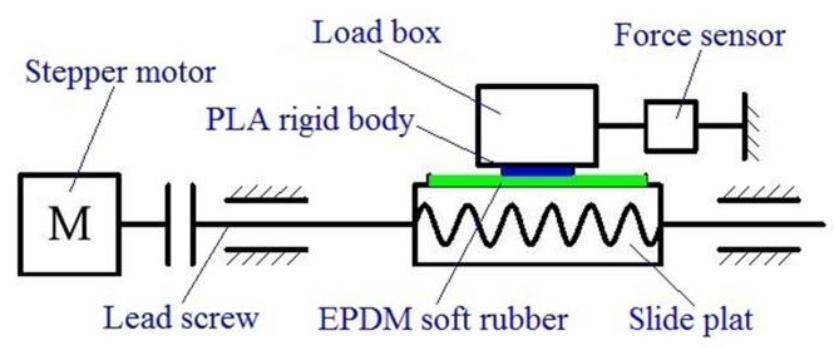

(a) Schematic diagram

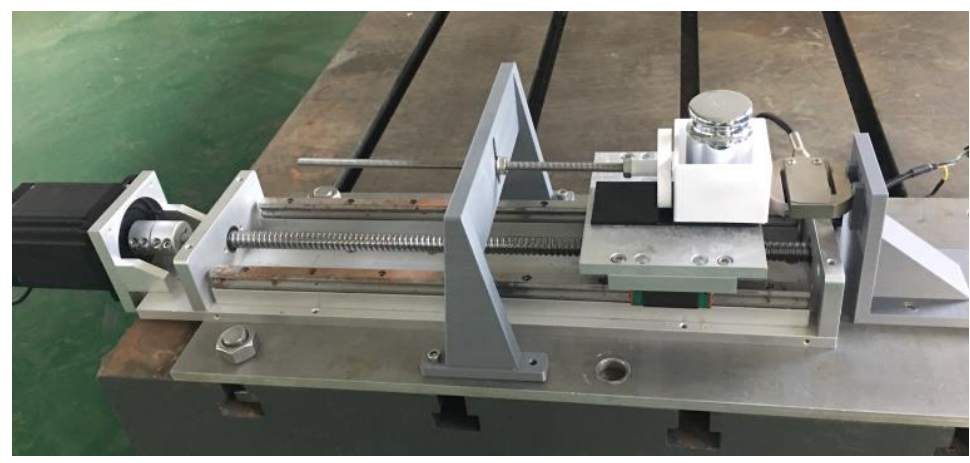

(b) Photo of the device

Fig. 2 The experimental device

\section{Results and discussion}

\subsection{Friction coefficient}

The formula for calculating the equivalent friction coefficient is as follows:

$$
\mu_{\mathrm{e}}=\frac{F_{\mathrm{f}}}{F_{\mathrm{n}}}
$$

where $F_{\mathrm{f}}$ is the apparent friction force $(\mathrm{N})$, and $F_{\mathrm{n}}$ is the applied normal load $(\mathrm{N})$. 
One of the experimental curves is shown in Fig. 3, which represents the relationship between friction force and time. $T_{1}$ is the stage from static to sliding, $T_{2}$ is the stage of stable sliding, and $T_{3}$ from sliding to static. In order to reduce the dynamic effects of velocity and strain changes, the values in stage $\mathrm{T}_{2}$ were averaged and used as the apparent friction force $F_{\mathrm{f}}$, and then the equivalent friction coefficients were calculated by Eq.(1).

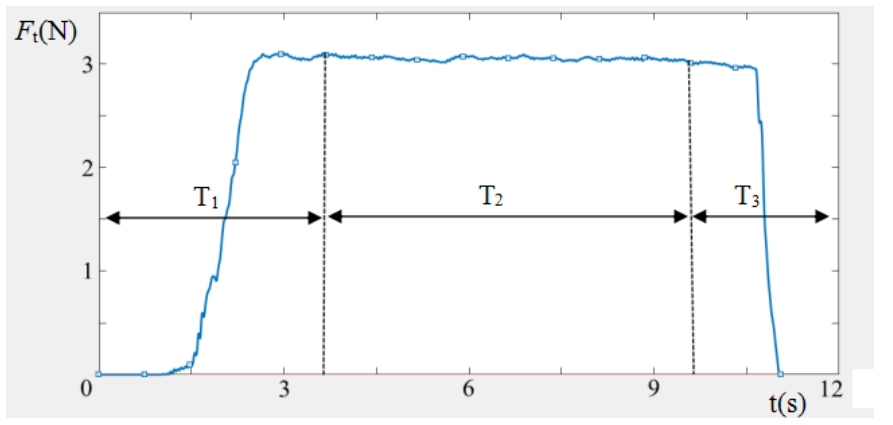

Fig. 3 A measured curve of friction force

The measured dependences of the equivalent friction coefficient on normal load are shown in Fig. 4. It can be seen that the friction coefficient between the spherical convex textured PLA rigid bodies and the EPDM soft rubber increases greatly with the increment of the normal load, and the rate of change slows down gradually when the load increases. Under the same applied load, the bigger the radius of the spherical convex texture, the smaller the equivalent friction coefficient between the friction couples. With the higher hardness of the rubber, the friction coefficient of non-textured surface changes slightly, the friction coefficient of the textured surface is smaller than that of the softer rubber.

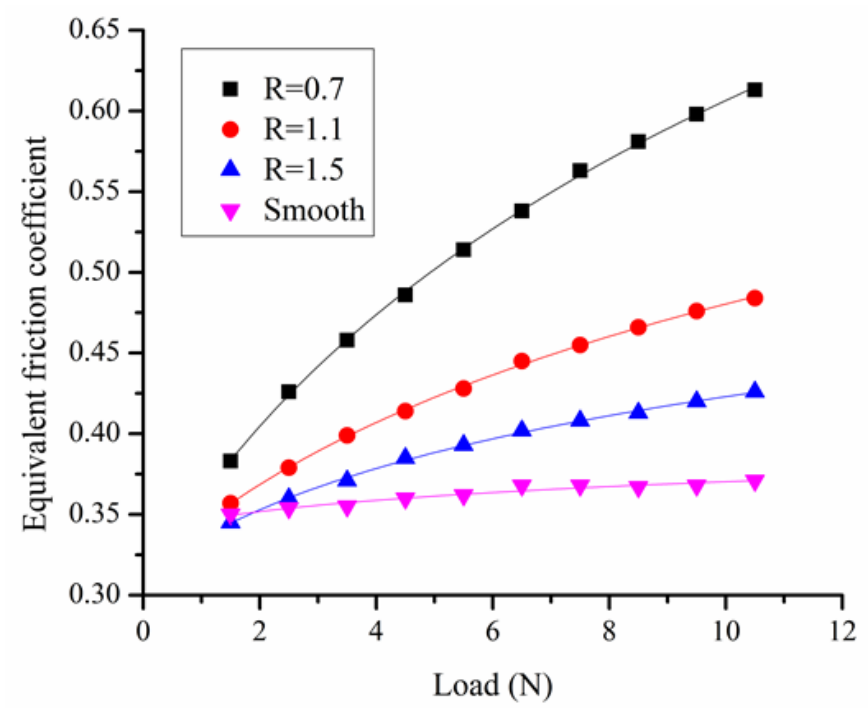

(a) The experimental results of sample hardness of 57HA 


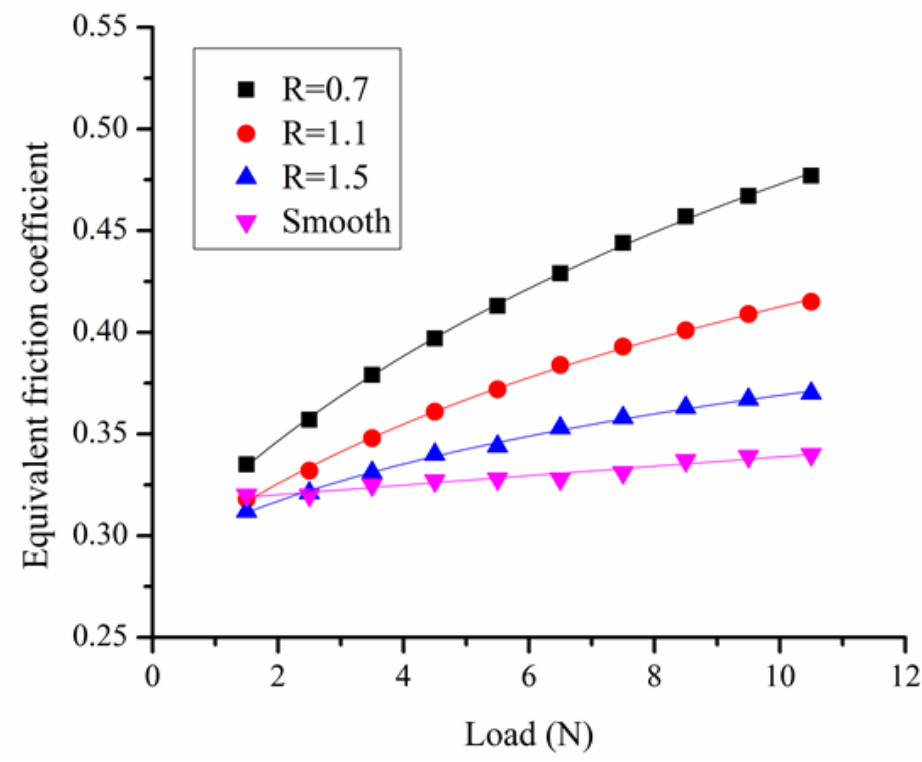

(b) The experimental results of sample hardness of 70HA

Fig. 4 The dependence of equivalent friction coefficient on normal load

It is also can be seen that the friction coefficients of the textured rigid bodies with textured spherical protrusion are less than that of smooth ones when the normal forces are small. The reason of this phenomenon is that adhesion between smooth rigid surface and the soft material is stronger than that of the textured couples. There should be non-contact area between the textured rigid surface and the rubber, which induces the adhesion effect became smaller.

\subsection{Discussion}

The rigid bodies do not deform when in contact with the soft rubber due to the hardness of PLA is far bigger than that of EPDM. The normal force can cause embedment of the spherical protrusion into the soft rubber, and resulting in large local deformation of the rubber. The deformation of the soft material with an embedded spherical protrusion is shown in Fig. 5. As the slide plat moves to the right side, the surface stress is mainly distributed in the left side of the spherical protrusion. At a point of the friction surface, the surface stress, consist of normal stress $f_{\mathrm{n}}$ and the frictional stress $\mu f_{\mathrm{n}}$, where $\mu$ is the friction coefficient at the point.

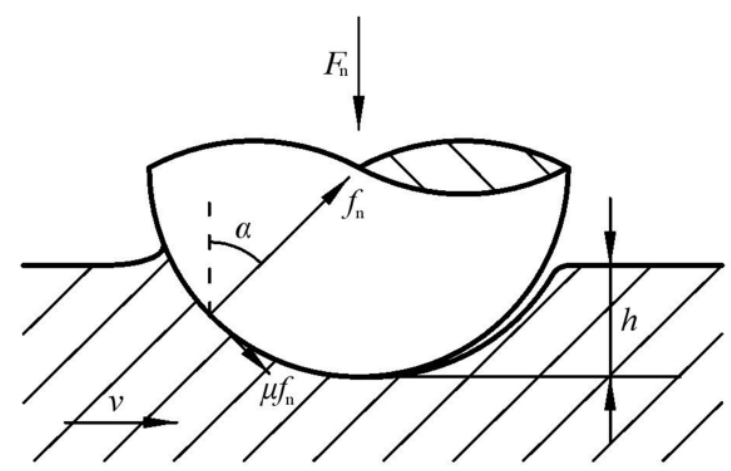

Fig. 5 Surface stress analysis of a spherical protrusion embedded in the rubber.

The distributions of the two kinds of surface stresses are all symmetric about the shown plane in Fig. 5. The component of resultant force of $f_{\mathrm{n}}$ perpendicular to the plane is 0 . The directions of the relative motion between the curved surfaces of the protrusion and the rubber are parallel to the plane basically. The two kinds of surface stresses can be decomposed to horizontal and vertical components. According to the principle of force equilibrium the normal force $F_{\mathrm{n}}$ and the apparent friction force $F_{\mathrm{f}}$ can be written as: 


$$
\begin{aligned}
& F_{\mathrm{n}}=\int_{\mathrm{s}}\left(f_{\mathrm{n}} \cos \alpha-\mu f_{\mathrm{n}} \sin \alpha\right) \mathrm{ds} \\
& F_{\mathrm{f}}=\int_{\mathrm{s}}\left(f_{\mathrm{n}} \sin \alpha+\mu f_{\mathrm{n}} \cos \alpha\right) \mathrm{ds}
\end{aligned}
$$

where $\alpha$ is the angle between the direction of $f_{\mathrm{n}}$ and the vertical direction.

Equation (3) indicates that apparent friction force consists of two components from different surface stresses. The component $\int_{\mathrm{s}} f_{\mathrm{n}} \sin \alpha \mathrm{ds}$ in Eq. (2) generates by the embedment of the spherical protrusion into the soft rubber, which can be called ploughing force. The other component generates by the relative sliding of the couple surfaces $\int_{\mathrm{s}} \mu f_{\mathrm{n}} \cos \alpha \mathrm{ds}$, which can be called sliding force (Wang, L. G., et al., 2007). It should be noticed that the normal stress $f_{\mathrm{n}}$ and the friction coefficient $\mu$ are all functions on the contacting area. The change of sliding force is far less than that of ploughing force with the change of the protrusion shapes, so it can be presumed that the friction coefficient $\mu$ is a constant on the contacting area, and then the expression of the equivalent friction coefficient can be derived from equations (1) and (2),

$$
\mu_{\mathrm{e}}=\frac{F_{\mathrm{f}}}{F_{\mathrm{n}}}=\mu+\frac{\left(1+\mu^{2}\right) \int_{\mathrm{s}} f_{\mathrm{n}} \sin \alpha \mathrm{ds}}{F_{\mathrm{n}}}
$$

It can be seen from Fig. 5that the range of $\alpha$ is $[0, \pi / 2]$, and then $\sin \alpha \geq 0$. The integral $\int_{\mathrm{s}} f_{\mathrm{n}} \sin \alpha \mathrm{ds}$ in Eq. 3 which is ploughing force is greater than 0 , therefore the equivalent friction coefficient $\mu_{\mathrm{e}}$ is always greater than $\mu$.

Equation (4) indicates that the ploughing force is the major factor changes the equivalent friction coefficient $\mu_{\mathrm{e}}$. The normal force changes $\mu_{\mathrm{e}}$ by changing normal surface stress $f_{\mathrm{n}}$ and contacting area $s$. The distribution of normal surface stress local $f_{\mathrm{n}}$ is difficult to determine because the deformation of the soft rubber is nonlinear large deformation, thus Eq.(4) cannot be used to predict $\mu_{\mathrm{e}}$ directly. It can be found from the previous study that surface stresses of the textured flexible friction couples are concentrated in a small area of the contact surfaces, and the places of the small area change with the change of normal force. Thus an effective angle $\alpha_{\mathrm{e}}$ of the contact area can be defined corresponding to the equivalent friction coefficient. The equivalent friction coefficient can be expressed by the effective angle as follow,

$$
\mu_{\mathrm{e}}=\frac{\sin \alpha_{\mathrm{e}}+\mu \cos \alpha_{\mathrm{e}}}{\cos \alpha_{\mathrm{e}}-\mu \sin \alpha_{\mathrm{e}}}
$$

where $\mu$ is the real friction coefficient of the contacting surfaces. The dependences of the equivalent friction coefficient on the effective angle are shown in Fig. 6. It can be seen that equivalent friction coefficient increases with increment of the real friction coefficient and the effective angle, and that the greater nonlinear influence on the equivalent friction coefficient is caused by the higher the real friction coefficient. 


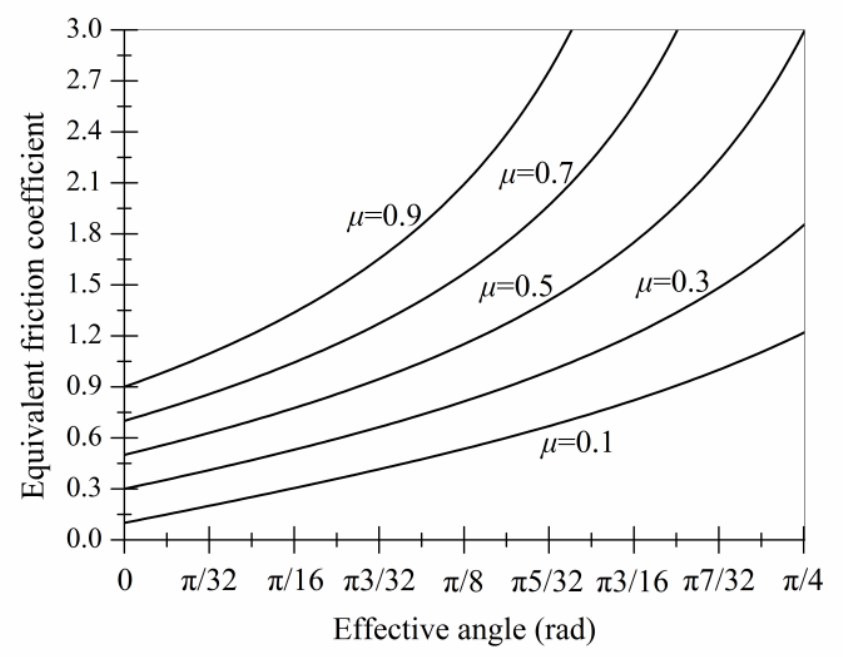

Fig. 6 The dependences of the equivalent friction coefficient on the equivalent angle

Different parameters of designed couples will have different frictional performances. For a certain couple, the effective angle $\alpha_{\mathrm{e}}$ increases with the increment of normal load $F_{\mathrm{n}}$, and the ploughing force increases to lead to an increment of equivalent friction coefficient $\mu_{\mathrm{e}}$. The radius $R$ of the spherical protrusions on the rigid body and the elastic modulus $E$ of the rubber, which is a hardness related parameter, have great effect on the embedment of protrusions into the rubber. The relationship between $F_{\mathrm{n}}$ and the depth $h$ of the pit is nonlinear and couples with parameters of $R, E$, etc. Hertzian contact theory can be used as a reference for calculating the depth although the theory is only applicable to linear small deformation. According to the solution of the Hertzian contact model of a sphere and an elastic half solid, the dependence of the applied load on the radius and the pit depth is $F_{\mathrm{n}}=\frac{4}{3} E^{*} R^{\frac{1}{2}} h^{\frac{3}{2}}$, where $R$ is the radius of the pit, and the equivalent elastic modulus is $E^{*}=\frac{E_{1} E_{2}}{\left(1-v_{1}^{2}\right) E_{2}+\left(1-v_{2}^{2}\right) E_{1}}$, in which $E_{1}$ and $E_{2}$ are elastic moduli, $v_{1}$ and $v_{2}$ are Poisson's ratios of the contact solids respectively. Since the elastic modulus of EPDM soft rubber is much smaller than that of PLA rigid body, so the equivalent elastic modulus can be simplified to $E^{*}=\frac{E}{1-v^{2}}$. For the textured flexible friction couple, the depth of the contacting pit can be approximately expressed by,

$$
h=\left[\frac{3 F_{n}\left(1-v^{2}\right)}{4 E \sqrt{R}}\right]^{\frac{2}{3}}
$$

where $E$ and $v$ are the elastic modulus and Poisson's ratio of the EPDM rubber respectively.

Although Eq. (6) can be used in case of linear elastic deformation of the soft rubber with a small force, a dimensionless variable can be derived,

$$
N_{\mathrm{ct}}=\left(1-v^{2}\right) \frac{F_{\mathrm{n}}}{E R^{2}}
$$

which can be called the embedment number for the contacting state of spherical protrusions.

The effective angle $\alpha_{\mathrm{e}}$ should be the function of the number of contacting $N_{\mathrm{ct}}$ in the frictional process of the textured flexible couples. The experimental results in Fig. 4 are recalculated by the transformation of Eq.(5), 


$$
\alpha_{\mathrm{e}}=\arctan \left(\frac{\mu_{\mathrm{e}}-\mu}{1+\mu_{\mathrm{e}} \mu}\right)
$$

in which $\mu_{\mathrm{e}}$ and $\mu$ are the friction coefficients with and without textures respectively. The corresponding $N_{\mathrm{ct}}$ are also calculated by Eq.(7). The calculating results are shown in Fig. 7 to display the relationship between $\alpha_{\mathrm{e}}$ and $N_{\text {ct. }}$ A fitting equation can be summarized as,

$$
\alpha_{\mathrm{e}}=0.12 \ln \left(1.07 N_{\mathrm{ct}}+1\right)
$$

The equivalent friction coefficient $\mu_{\mathrm{e}}$ of the designed textured flexible couples can be predicted by Eq. (5) and Eq. (9). The advantages of this method are the $\mu_{\mathrm{e}}$ can be calculated directly without complex nonlinear analysis of deformation and distribution of surface stresses, and the results are accurate.

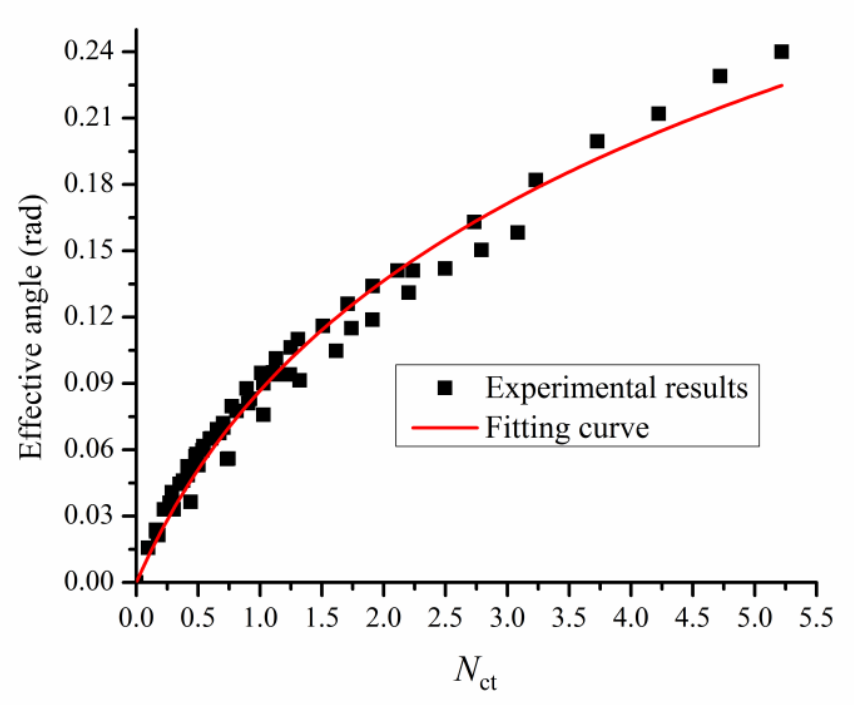

Fig. 7 Variation of effective angle with the embedment number

The polymer soft materials are hyperelastic and will not cause tearing failure under limited large strain. There is no obvious wear after the above experiments, so it can be considered that the ploughing effect caused by the protrusion is elastic. Therefore, the friction enhancement caused by this kind of protrusion is safe and practical. The large deformation of the soft rubber depends nonlinearly on the normal force, the surface stresses distributions of the contact area are complex, and the real friction coefficient is a distributing function on the contact area. Viscoelasticity of the rubber can affect the apparent friction force with large strain ratio during contacting process of the rubber and the protrusions because that the contacting state of surface textures of industrial friction couples are cyclic. All of the problems above will be studied in future work. Furthermore, the shape of the protrusions should be optimized to decrease the strain and stress of the rubber to prevent wear of the rubber.

\section{Conclusions}

The spherical convex texture was manufactured on the surface of rigid bodies (PLA) to make up flexible friction couples with the counterpart made of soft materials (EPDM). Experiments were carried out with low speed and dry friction condition. The following conclusions can be obtained according to the analyses of the experimental results:

(1) The apparent friction force consists of ploughing force and sliding friction force. The ploughing force induced by embedment of spherical protrusions into the soft material, and it is the main factor which changes the equivalent friction coefficient.

(2) The spherical convex texture can change the friction coefficient of the contact couples greatly. The equivalent friction coefficient increases with the increase of the applied load, but the rate of change gradually slows down. Under 
the same frictional condition, the friction couples with small radius of the spherical convex texture and low hardness of the soft material have large equivalent friction coefficients.

(3) The effective angle and the embedment number were proposed to simply the friction model and predict the equivalent friction coefficient. Equations were derived based on the experimental result, and can predict the friction coefficient very well.

\section{Acknowledgements}

The work was supported by the National Natural Science Foundation of China (No. 51575323).

\section{References}

Elwasli, F., Mzali, S., Zemzemi, F., Mkaddem, A. and Mezlini, S., Effects of initial surface topography and contact regimes on tribological behavior of AISI-52100/AA5083 materials' pair when reciprocating sliding. International Journal of Mechanical Sciences, Vol. 137(2018), pp.271-283.

Etsion, I., State of the art in laser surface texturing, Journal of tribology, Vol.127, No.1(2005), pp.248-253.

Hol, J., Alfaro, M. C., de Rooij, M. B. and Meinders, T., Advanced friction modeling for sheet metal forming. Wear Vol.286(2012), pp. 66-78.

Li, J., Liu, S., Yu, A. and Xiang, S., Effect of laser surface texture on CuSn6 bronze sliding against PTFE material under dry friction, Tribology International, Vol. 118(2018), pp. 37-45.

Menezes, P. L., Kailas, S. V. and Lovell, M. R., Role of surface texture, roughness, and hardness on friction during unidirectional sliding. Tribology letters, Vol.41, No.1(2011), pp.1-15.

Mielonen, K., Jiang, Y., Voyer J., Diem, A., Hillman, L., Suvanto, M. and Pakkanen, T. A., Sliding friction of hierarchically micro-micro textured polymer surfaces on ice. Cold Regions Science and Technology, Vol.163(2019), pp.8-18.

Miyanaga, N., Kishida, T. and Tomioka, J., Experimental investigation of load carrying capacity and frictional torque of dimpled parallel thrust bearings, Journal of Advanced Mechanical Design, Systems, and Manufacturing, Vol.14, No.3(2020), DOI: 10.1299/jamdsm.2020jamdsm0041.

Mukhopadhyay, A., SEM study of worn surface morphology of an indigenous 'EPDM' rubber. Polymer Testing, Vol.52(2016), pp.167-173.

Segu, D. Z. and Hwang, P., Friction control by multi-shape textured surface under pin-on-disc test, Tribology International, Vol.91(2015), pp.111-117.

Shimizu, M., Sawano, H., Yoshioka, H. and Shinno, H., Multi-dimensional assessment of nano/micro scale surface texture using laser speckle pattern analysis, Journal of Advanced Mechanical Design, Systems, and Manufacturing, Vol.9, No.1 (2015), DOI: 10.1299/jamdsm.2015jamdsm0011.

Varenberg, M., Halperin, G., Etsion, I., Different aspects of the role of wear debris in fretting wear. Wear, Vol.252, No. 11-12 (2002), pp.902-910.

Voyer, J., Jiang, Y., Pakkanen, T. A. and Diem, A., Adhesive friction and wear of micro-pillared polymers in dry contact, Polymer Testing, Vol.73(2019), pp. 258-267.

Wang, L. G., Sun, X. P. and Huang, Y., Friction analysis of microcosmic elastic-plastic contact for extrusion forming, Journal of materials processing technology, Vol. 187(2007), pp. 631-634.

Xiao, L. J., Sui, X. H., and Miao, D. J., Study on mechanics of driving drum with superelastic convexity surface covering-layer structure, Journal of Bionic Engineering, Vol. 5, No.1(2008), pp.60-65. 